\begin{tabular}{ccc}
\hline International Journal of Engineering \& Technology, $7(4.30)(2018) 397-402$ \\
SPC \\
Website www.sciencepubco.com/index.php/IJET \\
Research paper
\end{tabular}

\title{
An Automatic Transfusion Set for Accelerating Inoculation Process of Agarwood Artificial Inducer
}

\author{
Muhammad Nurrifat Roslee ${ }^{1}$, Siti Zarina Mohd Muji ${ }^{1 *}$, Balkis A Talip ${ }^{2}$, Norhayati Muhammad ${ }^{2}$, Norazlin Abdul- \\ lah$^{2}$, Rafidah Hamdan ${ }^{3}$, Suhaila Sari ${ }^{1}$, \\ ${ }^{1}$ Department of Computer Engineering, \\ Faculty of Electrical and Electronic Engineering, \\ Universiti Tun Hussein Onn Malaysia, 86400 Parit Raja, Batu Pahat, Johor \\ ${ }^{2}$ Department of Technology and Natural Resources, \\ Faculty of Applied Sciences and Technology, \\ Universiti Tun Hussein Onn Malaysia, KM1 Jalan Panchor, 84000 Pagoh, Muar, Johor \\ ${ }^{2}$ Department of Water and Environmental Engineering, \\ Faculty of Civil and Environmental Engineering, \\ Universiti Tun Hussein Onn Malaysia, 86400 Parit Raja, Batu Pahat, Johor \\ *Corresponding authorE-mail: szarina@uthm.edu.my
}

\begin{abstract}
The formation of agarwood resin can be accelerated by natural or artificial intervention of Aquilaria tree. Many planters developed varies inoculant or inducer in order to accelerate the formation of agarwood. Aquilaria trees require more than 20 years to form natural agarwood formation naturally, thus it becomes impractical especially for commercial purposes. On the other hand, the production of varies booster commonly unequipped with standard inoculating apparatus. The existing agarwood-inducing techniques require longer time to complete the inoculation process for each tree. Therefore, this study aims to develop a novel automated transfusion set for facilitating the inoculation process of Aquilaria tree. An automatic transfusion is equipped with magnetic sensor to monitor the inoculants volume. The automated transfusion set operated by $12 \mathrm{~V}$ water pump that controlled by Arduino controller to give a constant pressure during inoculation process. The infusion of inoculants also controlled by Arduino controller with aids of magnetic sensor. Hence, the total volume of inoculants being infused inside the Aquilaria tree can be monitored. The result for the automated transfusion is indeed reduces the time of inoculation process which is roughly $70 \mathrm{ml}$ in 2 hours compared to the conventional techniques (whole tree inoculation and syringes inoculation) by 2 hours for $50 \mathrm{ml}$. Thus, this invention is potentially benefitting the planters to reduce time and laborious particularly in inoculating liquid inducers or inoculants.
\end{abstract}

Keywords: Agarwood; Arduino; Inoculants; Magnetic Sensor; Transfusion Set

\section{Introduction}

Recent years, the use of agarwood oil in Malaysia has been significantly increase and also the extraction has become popular and widely applied [1]. Agarwood has been renowned as the oil-based perfume in the middle-eastern and many researchers have claimed that agarwood oil has variety usage such as in traditional medical, in religious and traditional ceremony and also as the key ingredient in tea [2]. Agarwood production occurs naturally only $10 \%$ of Aquilaria tree [3]. To reduce the dependence on the wild agarwood, Aquilaria species have been cultivated on a large scale particularly in Johor, Malaysia. Asia Plantation Capital has invested approximately 50 million US Dollar and planted a total 13,400 Aquilaria species in Batu Pahat as phase one [4].

Agarwood is the resin that produced from the wounded Aquilaria tree. This has made the tree valuable and expensive by injecting the inoculants into the xylem tissue of the tree. Literally, the agarwood is formed and spread to whole part of tree due to transpiration process of water occurred in xylem tissue. Thus, the agarwood production will increase gradually [5]. However, to induce the inoculants or chemical substances inside the Xylem of the tree, it takes an approximately 2 hours for a medium size of trunk. Furthermore, this process requires a fully-supervised by human throughout the entire process. Hence, it is very laborious and stressful. The demand of agarwood products increases gradually hence, the inoculation process become crucial and the need of manpower to accomplish this task is essential. Hence, agarwood production business and maintenance are indeed costly. Thus, the development of transfusion set aims to involve minimum human supervision and less time needed for the process to be completed.

\section{Related Review}

Historically, in Song dynasty farmers induced the formation of agarwood by destructive methods such as axing, severe bark removal and nailing [6]. Figure 1 shows the different techniques and diagrammatic representation of induction practices which usually applied by researcher and farmer.

Figure 1 (a) shows the agarwood production without any induction or injury happened but resin may be formed naturally by natural stresses in very long time (20-30 years). Figure 1 (b) shows an artificial induction by drilling the trunk, root and large branches 
and agarwood produces within at least 12 months. Figure 1 (c) shows a technique by drilling pores and kept it open to attract insect and ignite the infection process. This process is fully assisted by sugary syrup and PVC tubes installed inside the tree's holes preventing healing process within the tree. Figure 1 (d) shows the inoculants induction using syringe that being used to facilitate batch and continuous inoculation and induction. A severe bark removal was applied in some region and this technique lead to the core tissue affection as shown in Figure1 (e). Figure1 (f) shows the certain area of trunk of the tree was dug to make into hole for hatching ants and snails and eventually will lead to the formation of agarwood.

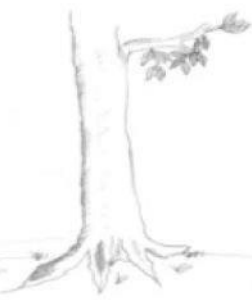

(a)

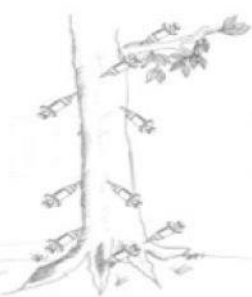

(d)

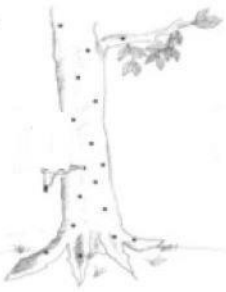

(b)

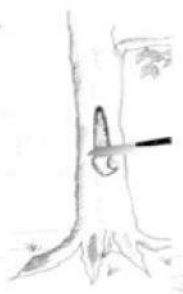

(e)

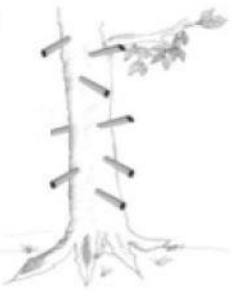

(c)

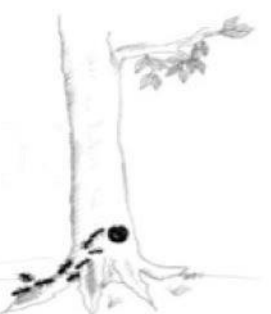

(f)
Fig. 1: Illustration of induction methods commonly used for agarwood production: a) No injury or induction; b) Artificial induction drilling the stem; c) Open drilled pore filled with sugary syrup; d) Syringe with inoculants and inducers; e) Severe bark removing; f) Dug hole to attract insect [7]

However, some techniques yield poor-quality agarwood and low in agarwood production. Recent years, the technique use for in ducing the inoculants into the trunk of Aquilaria sp. has evolved and each have showed different advantages and abilities. According to Chong et al. 2015 [5], several holes which end at the xylem of the tree should be drilled in form of spiral shape to maximize the production of agarwood inside the trunk of the tree as shown in Figure2. This study has shown the significant production of agarwood as compared to the yield from other techniques.

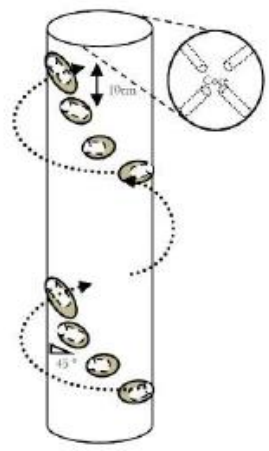

Fig. 2: The holes drilled in spiral shaped on Aquilaria Trees [5].

A study by Yangyang Liu, 2013, [6] has listed four techniques to speed up the formation of agarwood which involve whole-tree agarwood-inducing technique (Agar-wit), partly-trunk-pruning method (PTP), burning-chisel-drilling method (BCD) and fungiinoculation (FI) (Figure3).

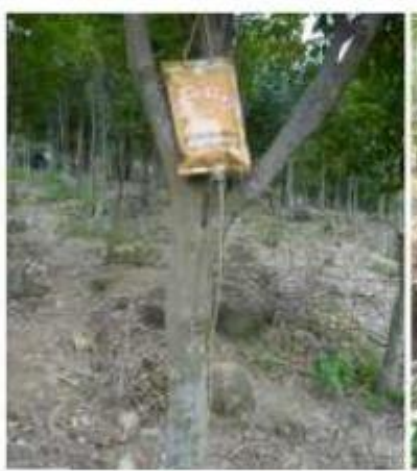

(a)

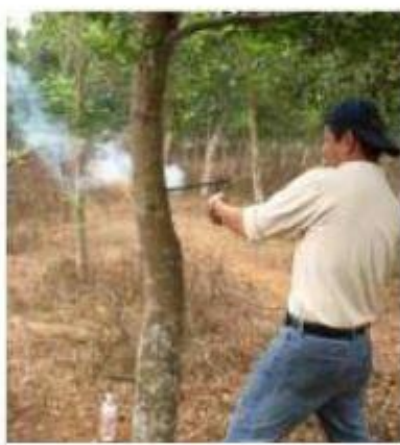

(c)

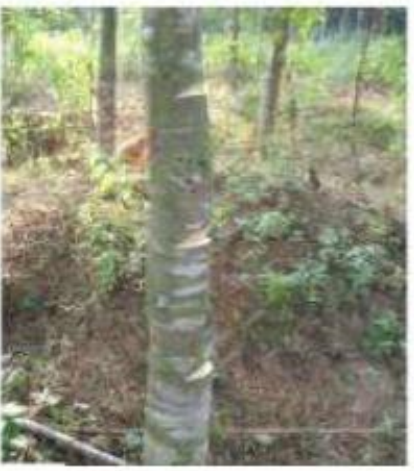

(b)

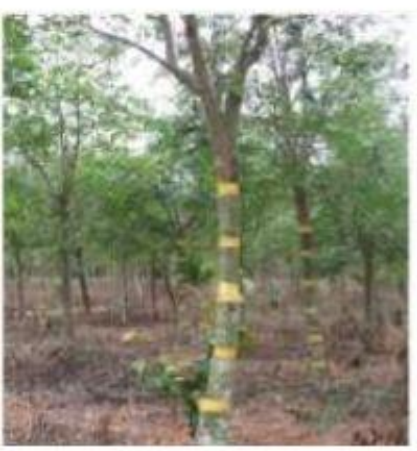

(d)
Fig. 3: The four agarwood-inducing techniques. (a) Agar-Wit (b) PTP (c) BCD (d) FI [6]

Maoxun Yang, 2014 had developed a new device called Modified Kit for infusion technique in order to improve the infusion rate of efficiency [8]. This Modified Kit consists of five inducers and procedures which are transfusion needle and vessel, inducer and procedure for primary and secondary process of agarwoodinducing technique.

Figure 4 shows a needle used in Modified Kit. This needle was modified accordingly with the process of agarwood-inducing technique suitability. The sunken-style equipped at the tip of needle functions as to make sure the inoculants inducing-process is smoothly performed with any equipment failure. For examples, the syringe is pulled out of the tree trunk due to constant pressure from the syringe. A liquid storage cavity is formed between the fluid outlets and hole walls when the needle being inserted into the trunks, which completely prevents the fluid outlets from being blocked, and the fluid from oozing out of the trunks, thus improves the infusion efficiency.

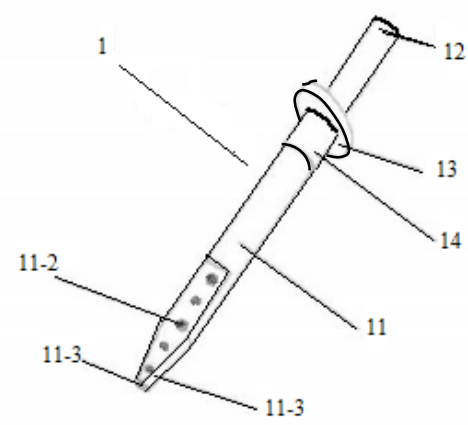

Fig. 4: The plant transfusion needle in Modified Kit: 1) Needle; 11) Needle body; 11-1) Fluid outlet; 11-2) Drain hole; 11-3) Needle tip; 12) Connector; 13) Projecting shoulder; 14) Squeezing part [8].

Figure 5 shows a design of Modified Kit of transfusion vessel. The vessel is divided into two or more independent chambers, 
each connecting to the transfusion tube and needle. If any of the needle or tube is clogged, the water level in the connected chamber will stop lowering, prompting the clog to be detected and handled in time, thus ensuring an effective infusion. This equipment has developed to increase the efficiency and smoothen the agarwood-inducing technique process.

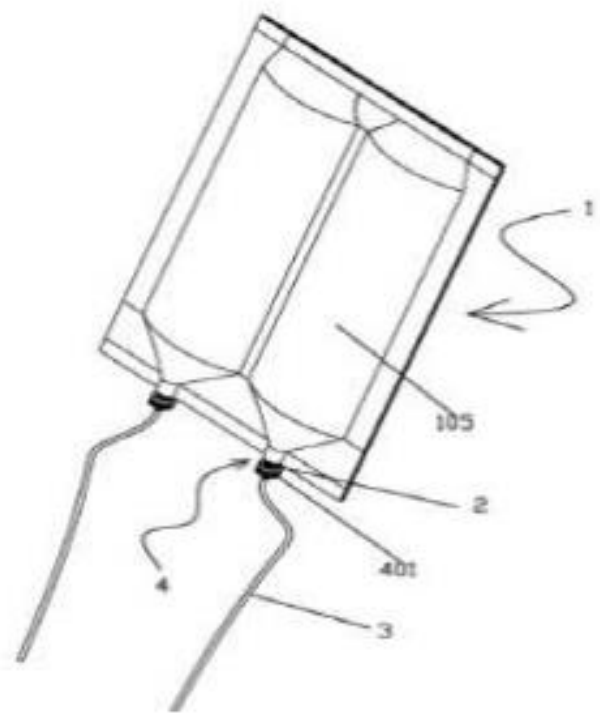

Fig. 5: The transfusion vessel in Modified Kit: (1) Vessel body; (105) Plastic substrate; (2) Vessel spout; (3) Infusion tube; (4) Connective structure; (401) Spout cover [8].

Based on the review, the best way to produce the prototype is by using modern technique because it yields good grade of agarwood and also the formation of agarwood is faster and greater in size and density. This project uses the automatic system by Arduino controller and water pump that expected to reduce the operation time. The wall plug that connected to the water pump pipe used as function to induce the inoculants. The wall plug substitutes the use of syringes as inducer dispenser. This is because when pressure is applied on the system constantly, eventually the syringe will pull out as reaction to constant pressure and decreases the efficiency of inoculation process.

\section{Methodology}

This prototype is mainly controlled by microcontroller Arduino. The Arduino controls the movement of $12 \mathrm{~V}$ direct current water pump motor and monitor the volume of inoculants within the container using magnetic sensor as water level detection. The water pump is used to keep pressuring the inoculants into the tree trunk. $12 \mathrm{~V}$ relay circuit used to control the water pump motor from $12 \mathrm{~V}$ direct current (DC) source battery. The Arduino microcontroller is then connected with GSM module for monitoring the whole process to be completed.

Figure 3.1 shows the flow chart of transfusion set from the voltage supply until it reached to the end user. Battery is used to power the whole system or to start the process of the system by applying $12 \mathrm{~V}$ DC supply towards the whole system. Then it powered the Arduino board with $5 \mathrm{~V}$, this microcontroller is the main part of this transfusion set whereby it controls all activities inside this hardware. When the inoculants poured inside the container approximately $250 \mathrm{ml}$, the magnetic sensor detects the level of the liquids. This Magnetic sensor is attached to the wall of inoculants container. This sensor activates DC water pump to pump out all the inoculants inside the Aquilaria tree. Whenever of the inoculants were entirely pumped out, the magnetic sensed, then stop the operation of water pump.

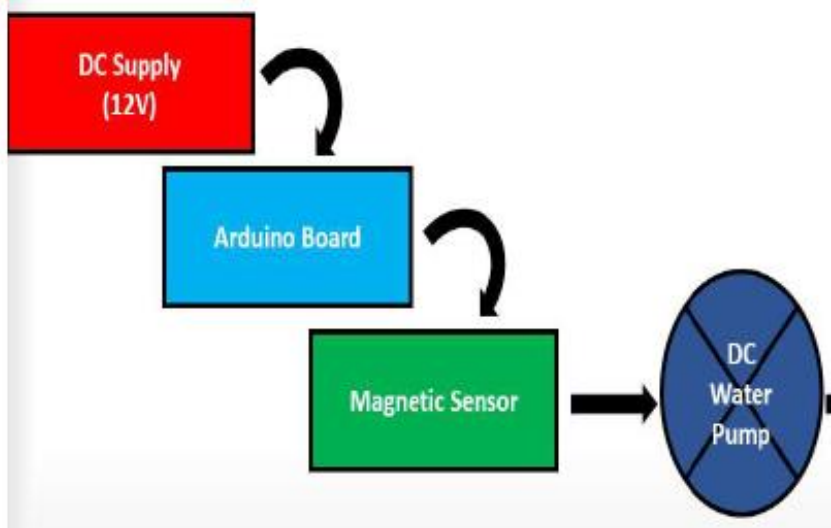

Fig. 6: The flowchart of the transfusion set system.

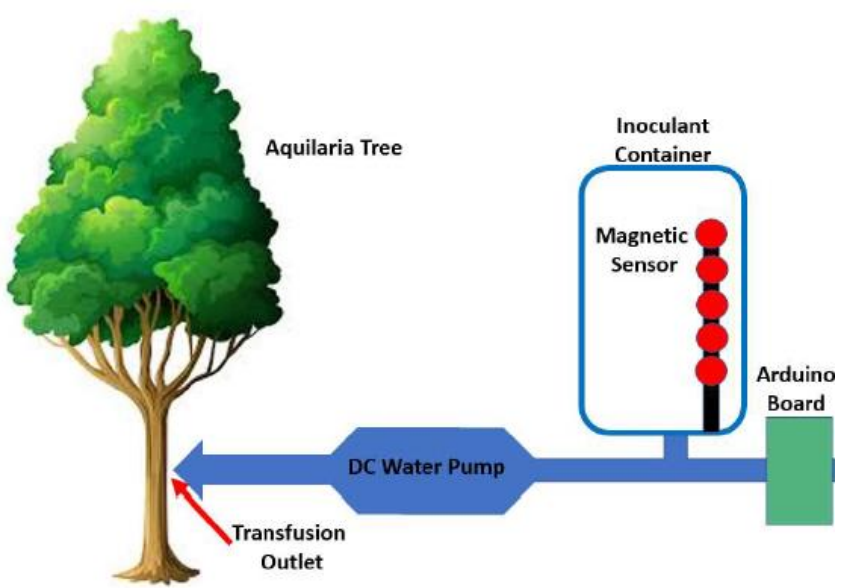

Fig. 7: The prototype design and agarwood-inducing technique:

Based on Figure7, the equipments stated have different functional role as described in section 31-3.6.

\subsection{Transfusion outlet}

The transfusion outlet is the combination between wall plug and end of water pump pipe that will be placed into the Aquilaria Tree hole. Polytetrafluoroethylene (PTFE) tape is used to avoid leaking between these two connections. The wall plug was cut at 45 degree for positioning in the tree hole.

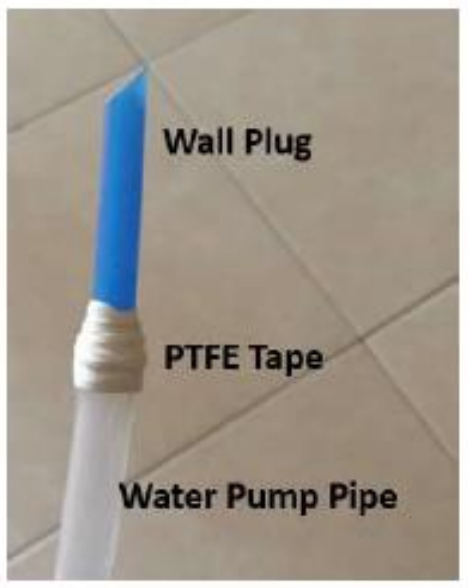

Fig. 8: The transfusion outlet.

\subsection{Inoculants container}

The function of inoculants container as shown in Fig 9 is to place the inoculants. It is completely sealed with only one way out which then connected with inlet of DC water pump. The maximum volume of this container is $500 \mathrm{ml}$. It has to be sealed in 
order to avoid insects or any animals that may intervened the inoculation process. The container is convenient and practical to be used in agarwood-inducing process. Magnetic sensor was equipped inside this container to indicate whether the liquid already completely insert or not.

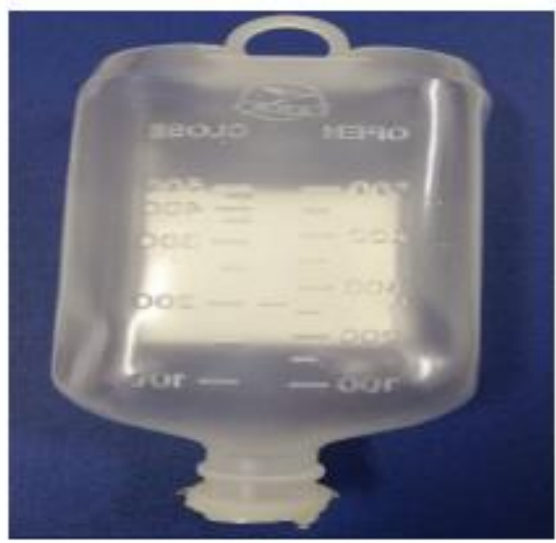

Fig. 9: Inoculants container.

\subsection{Arduino controller}

All of system need controller to control every single movement or function that happened. Arduino controller is used to communicate with input and output peripherals in making the systems works. This prototype research has used Arduino Uno controller to control the movement of water pump motor. It turn-on the water pump whenever there is trigger and turn-off whenever the water level sensor detected a desired level of inoculants reduced and thus indicated the process completed.

\subsection{DC water pump}

The usage of water pump in this prototype is to give a constant amount of pressure within desired time. A $12 \mathrm{~V}$ DC water pump with power range from $0.5 \mathrm{Watt}$ to $5 \mathrm{Watt}$ and able to pump out of maximum flow 350 litres/hours. This water pump is waterproof hence, it is very practical due to process of agarwood-inducing is approximately 2 hours and exposed to natural weather. This water pump will pump out the inoculants from the container to the holemade of trunk of the tree. This process will keep running until the specific volume of inoculants has been induced into the tree trunk is diffused.

\subsection{Reed switch (magnetic sensor)}

Reed switch is an electrical switch when there is magnetic field of a magnet has been applied. Reed switch has two flexible magnetizable metal reed that separated by a very small gap when switch is open. When there is magnetic field either from electromagnetic or permanent magnetic it would cause the both metal reeds attract to each other [9]. When magnetic field is absent the metal reeds will back to its normal position which is normally open circuit.

\subsection{Sensor design (water level detection)}

Reed switch has been used as part of magnetic sensor to detect the water level sensor. Fig 10 shows the proposed design of the sensor and application of magnetic sensor on detecting the water level. The operation is that the reed switch is placed in 5 different levels of height which indicate the volume of inoculants inside the container. Then, permanent magnet is attached to the Styrofoam to make it float. As the inoculant volume decreased, the magnet also decreased in height. This operation can detect the level of inoculant at all time

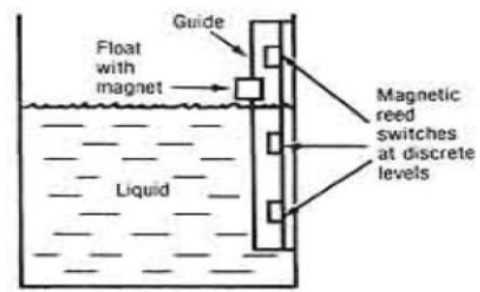

Fig. 10: The application of magnetic sensor on water level detection [10]

All of the reed switch is connected to the Arduino digital pin. As the reed switch is always open condition, it indicates 0 and when it is closed the value is equal to 1 . Whenever the inoculants are filled at maximum level, the highest level of reed switch detect the magnetic field from the floating permanent magnet as shown in Fig 11. Then as the volume decreased, reed switch will detect the movement of permanent magnet to show the current inoculants level.

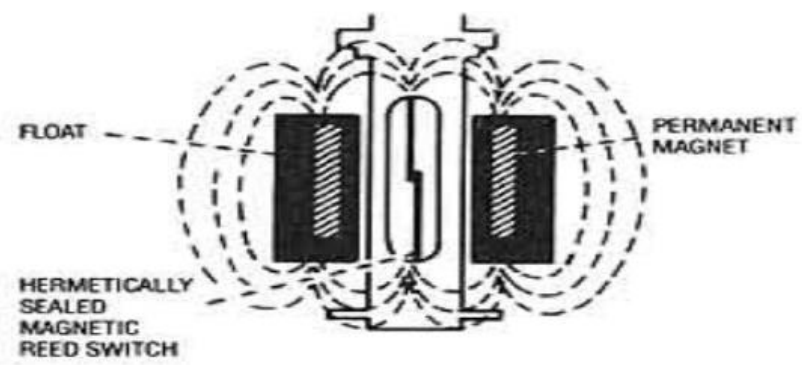

Fig. 11: The concept of magnetic sensor between reed switch and permanent magnet.

\subsection{Preparation of inoculation process}

Inoculation process is the process whereby the inoculants being infused inside the Aquilaria tree by the aid of an automated transfusion set. However, before this process can be start there are numbers of procedure to follow which are:

1) Drilling process. Make hole to the trunk of Aquilaria tree so that the transfusion outlet able to stick into the tree trunk.

2) Insert the transfusion outlet. Make sure the plug wall tip is cut in 45 degrees shape to prevent the transfusion outlet fall down.

3) Fill in the inoculants inside the inoculant container. Close the container after it filled to prevent any insect or animal attracted to it.

4) Switch on the hardware to initiate the process of inoculation.

\subsection{Hardware development}

Hardware development is crucial part in designing the hardware project. The outcome of the design must be applicable and suitable to the environment where it could be applied. Fig 12 shows the block diagram of the hardware from the power supply until to the end user. This product will be applied in the remote area where normally all the Aquilaria trees are planted. The probability of that particular area to have electricity supply is low. Hence, the use of portable and rechargeable battery can solve this problem. In fact, it is more gematrically suitable caused transfusion process required lot of movement to complete one whole plantation. Relay circuit is used as to control the motor of the water pump since direct current is applied. This is due to the DC current cannot directly control the motor. For DC water pump, it is used for generating the pressure to pump out the inoculants into the Aquilaria tree. This is essential when compared to previous method without using water pump, the whole process of transfusion takes 1 whole day and more. By using this, the time required to complete the whole process can be reduced.

Magnetic sensor is used to monitor the level of inoculants throughout the transfusion process. Magnetic sensor consists of two components which are permanent magnet and reed switch. 
Both magnetic sensor and water pump are being controlled by Arduino. This is where all the process is being coordinated by the coding until the whole process is done.

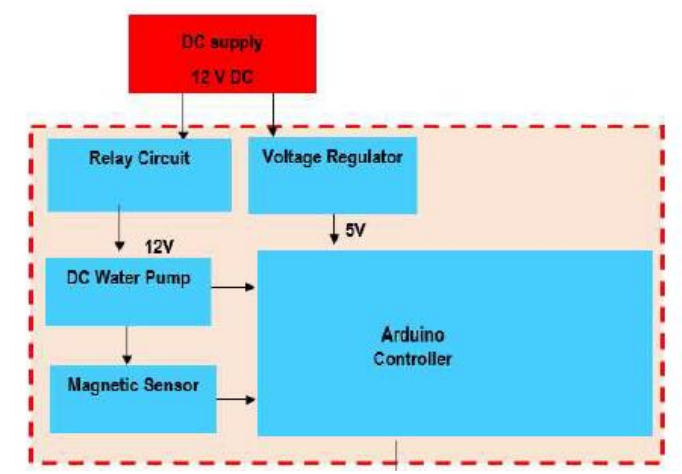

Fig. 12: The block diagram of the hardware.

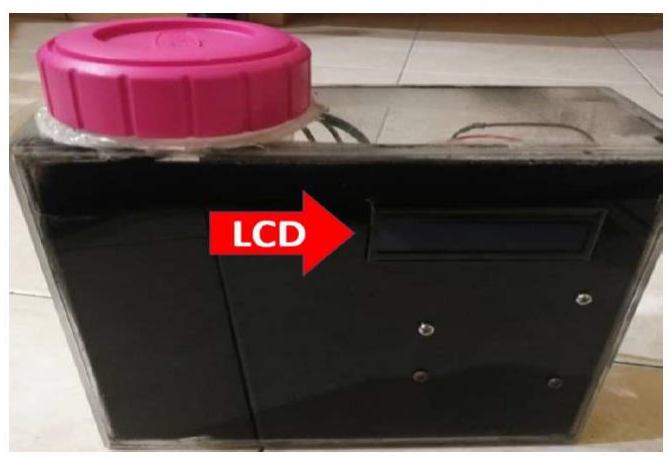

Fig. 13: The front view of the hardware

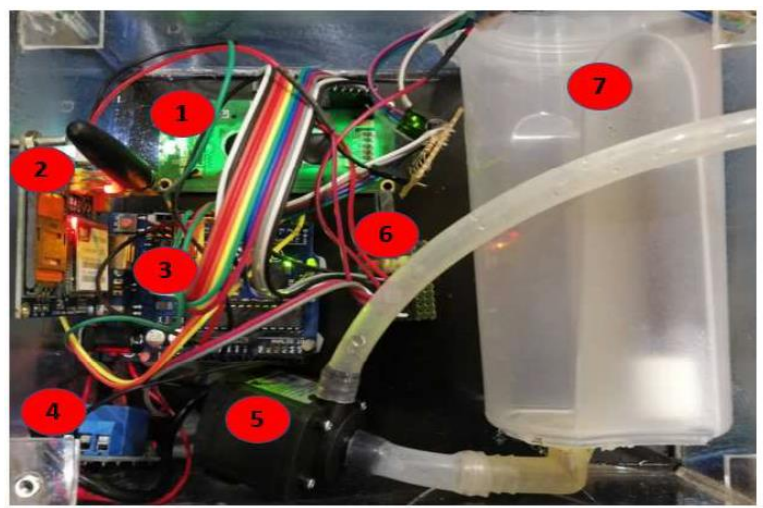

Fig. 14: The back view of the hardware; 1) LCD; 2) GSM Module; 3) Arduino Board; 4) Relay Circuit; 5) Water Pump; 6) Variable Resistor; 7) Inoculants Container.

The hardware is then being connected to the DC supply to see whether the overall connection of the circuit and the components are good. As shown in Figure 15 the hardware is fully connected and functioning as predicted.

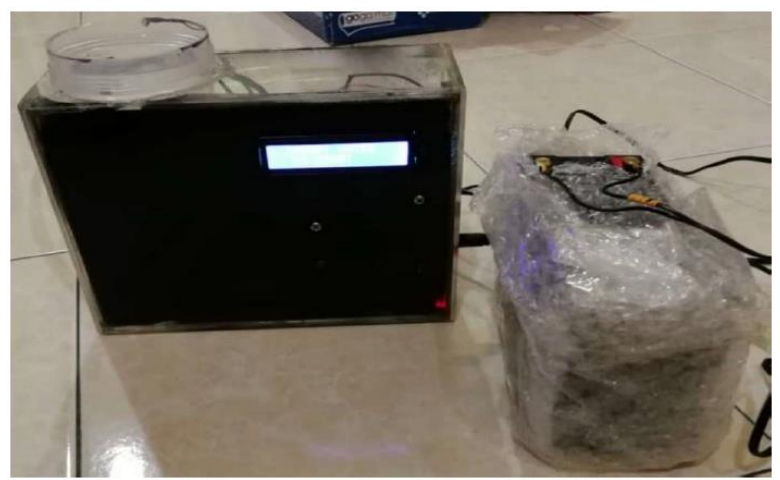

Fig. 15: The prototype of automated transfusion set for agarwood inducer

\section{Result and Analysis}

\subsection{The functionality of an automated transfusion set}

Figure 16 (a) until 16(d) shows different level of liquid inside the inoculant container. The magnetic sensor attached to the inoculant container is able to detect and sense the current level of inoculant with aids of floating permanent magnet.

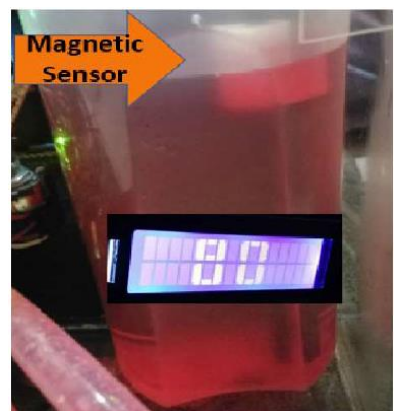

(a)

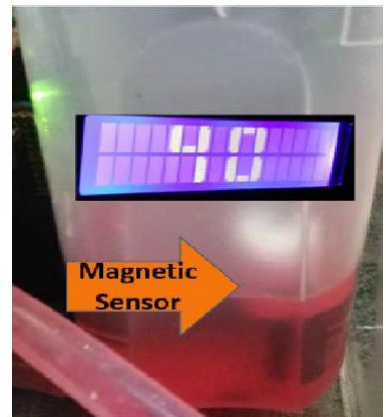

(c)

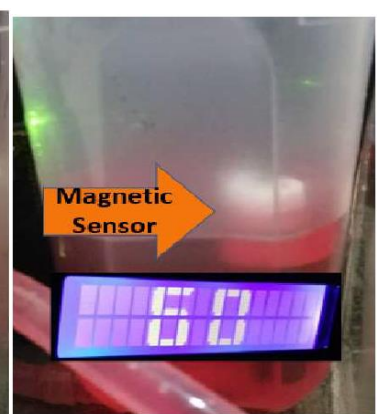

(b)

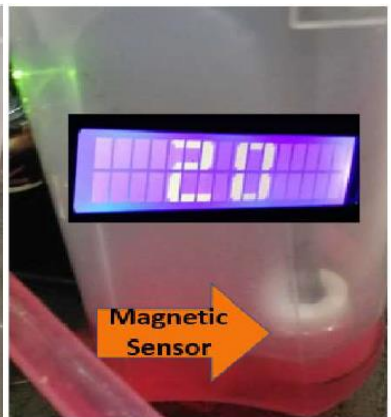

(d)
Fig. 16: The volume of inoculants that sensed by the magnetic sensor, (a) $80 \%$ level (b) $60 \%$ level (c) $40 \%$ level (d) $20 \%$ level

The results have shown that the components involved in detecting the current level of inoculant were successfully operated. It is indicated that the Arduino coding was accurately programmed as well as the connection between the hardware is correctly constructed and functioned.

\subsection{The application of hardware: inoculation process of Aquilaria Tree}

This study aims to reduce the operation time for inoculation process and reduces the laborious cost. Previously, the manual technique takes at least 2 hours to insert a small volume of inoculant. Thus, it is expected that this automated transfusion set can reduces both operation time and laborious cost. The prototype of agarwood automated transfusion set was tested on Aquilaria tree as shown in Figure 17 and Figure 18.

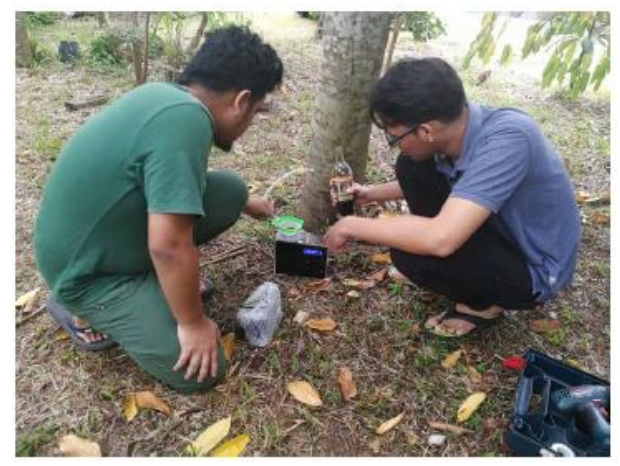

Fig. 17: The preparation before the inoculation process begin. 


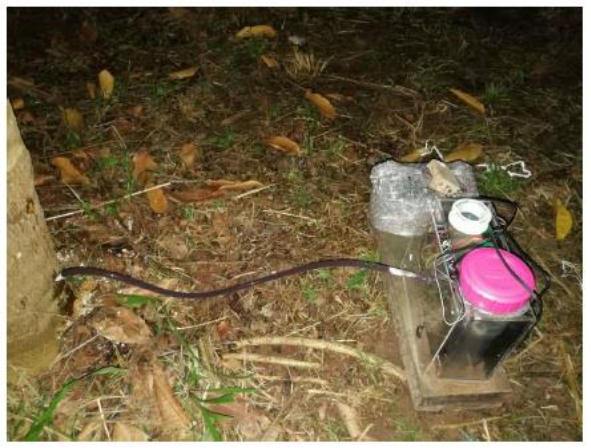

Fig. 18: The inoculation process.

Figure 19 (a) and (b) shows the two different volume of inoculant before and after the inoculation process. There were slightly changes in volume of inoculant which indicate this concept is applicable. However, the volume that has been transfused into the Aquilaria tree was about $70 \mathrm{ml}$ for less than 2 hours. There were few factors that may contribute to the slow motion of transfusion including the life span of the batteries and type of inoculants. The batteries can degrade due to its life span thus deteriorate its efficiency to supply constant voltage resulting in decreasing of voltage supply. Meanwhile, the inoculation solution must be diluted sufficiently in order to avoid coagulation and sedimentation in the container or tube.

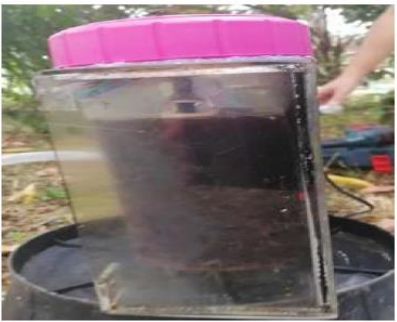

(a)

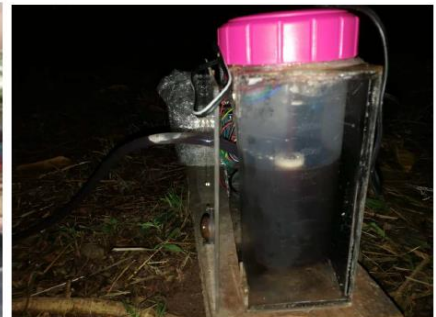

(b)
Fig. 19: The level of volume of innoculant (a) The volume of inoculants before inoculation process begin (b)The volume of inoculants after the inoculation process begun.

\section{Acknowledgement}

This research was conducted with financial support by Geran Pembangunan Produk (GPP) Vot number B080.

\section{References}

[1] Rashid AYZ (2001), Tapping The Wealth From Karas Tree. Forest Research Institute Malaysia.

[2] Abdul MFKH (2013), Extraction of Essential Oil from Biologically Inoculated Agarwood. 2013 IEEE Business Engineering and Industrial Applications Colloquium (BEIAC).

[3] Nurlaila IMH (2015), A review on Agarwood and Its Quality Determination . 2015 IEEE 6th Control and System Graduate Research Colloquium 1.

[4] Asia Plantation Capital Berhad Continues to Invest in Malaysia (2016, April 27). Retrieved from Asia Plantation Capital https://www.asiaplantationcapital.com/2016/04/27/asia-plantationcapital-berhadcontinues- invest-malaysia.

[5] Chong SOR (2015), Agarwood Inducement Technology: A Method for Producing Oil Grade Agarwood in Cultivated Aquilaria malaccenses Lamk. Agrotechnology and Bioscience Division, Malaysian Nuclear Agency (Nuclear Malaysia).

[6] Yangyang LHC (2013), Whole-tree Agarwood-Inducing Technique: An Afficient Novel Tecnique for Producing High-Quality Agarwood in Cultivated Aquilaria Sinensis Trees. Molecules, 30863106.

[7] Selina AMT (2013), Agardwood Production- A Multidisciplinary Field To Be Explored In Bangladesh. International Journal of Pharmaceutical and Life Science 22.
[8] Maoxun YHF (2014), Modified Transfusion Devices, Inducer, and Procedure for Agarwood-inducing by Infusion Techniques. Journal of Chemical and Pharmaceutical Research, 2566-2571.

[9] Reed Switch (2018, March 15). Retrieved from Wikipedia : https://en.wikipedia.org/wiki/Reed_switch.

[10] Point-Contact Level Sensors. (2008, August 18). Retrieved from Industrial Electronics: http://www.industrialelectronics. com/DAQ/industrial_electronics/input_devices_sensors_transducer s_transmitters_measurement/Point-Contact-Level Sensors.html 